\title{
Psicooncología
}

ISSN: $1696-7240$

https://dx.doi.org/10.5209/psic.77760

\section{¿Cómo explico que tengo cáncer a las personas mayores de mi familia?: díptico de soporte para pacientes recién diagnosticados}

\section{[en] How do I explain that I have cancer to the elderly in my family?: Support brochure for newly diagnosed patients}

Sònia Fuentes Sanmartín ${ }^{1 *}$; Milagros Barez Villoria²; Mercé Riquelme Olivares ${ }^{3}$; Sabelia Seguro Calabozo ${ }^{4}$

Recibido: 17 de septiembre de 202o / 2 de febrero de 2021

Cómo citar: Fuentes Sanmartín S, Barez Villoria M, Riquelme Olivares M, Seguro Calabozo S. ¿Cómo explico que tengo cáncer a las personas mayores de mi familia?: díptico de soporte para pacientes recién diagnosticados. Psicooncología 2021; 18: 387-391, https://dx.doi.org/10.5209/psic.77760

Comunicar las malas noticias relacionadas con la aparición de la enfermedad supone para muchos pacientes oncológicos una situación de gran dificultad y sufrimiento. A menudo, el diagnóstico actúa como un elemento generador de crisis en el seno del núcleo familiar. La enfermedad puede ser vivida como una amenaza que distorsiona las rutinas familiares y provoca dificultades en la adaptación a esta nueva situación de pérdida. Los significados otorgados a la enfermedad condicionan notablemente el tipo de estrategia de afrontamiento a adoptar y suelen generar alteraciones en la comunicación familiar ${ }^{(1)}$.

Muchos de las personas que se encuentran en esta situación de enfermedad tienen padres muy mayores y con frecuencia plantean a los profesionales que componen el

1 Sònia Fuentes Sanmartín. Unidad de Psicooncología Instituto Catalán de Oncología,. Hospital Universitario GermansTrias i Pujol. Badalona, Barcelona España

E-mail: sfuentes@iconcologia.net

2 Milagros Barez Villoria Unidad de Psicooncología Instituto Catalán de Oncología,. Hospital Universitario GermansTrias i Pujol. Badalona, Barcelona España

E-mail: mriquelme@iconcologia.net

3 Mercé Riquelme Olivares. Unidad de Trabajo Social Instituto Catalán de Oncología, Badalona. Hospital Universitario Germans Trias i Pujol. Badalona, Barcelona España

E-mail:mbarez@iconcologia.net

4 Sabelia Seguro Calabozo. Unidad de Trabajo Social Instituto Catalán de Oncología, Badalona. Hospital Universitario Germans Trias i Pujo. Badalona, Barcelona España 1.

E-mail: sseguro@iconcologia.net

* Dirección de correspondencia: Sònia Fuentes Sanmartín. Unidad de Psicooncología. Instituto Catalán de Oncología -Badalona. Hospital Universitario Germans Trias i Pujol. Carretera del Canyet, s/n.08916 - Badalona. España. E-mail: sfuentes@iconcologia.net 
equipo de cuidados oncológicos, cuestiones sobre cómo hablar sobre la enfermedad a sus padres ya ancianos.

Como ya hemos descrito en anteriores publicaciones relacionadas con el tema de la comunicación intrafamiliar, detectamos cada vez con mayor frecuencia, por parte de las personas enfermas, protección informativa hacia sus hijos menores ${ }^{(2-4)}$, pero también hacia las personas de edad avanzada que forman parte de su familia (madres, padres, suegros, etc.).

La bibliografía al respecto, en el campo de las enfermedades oncológicas, es muy limitada, pero los estudios revisados concluyen que ocultar la información con la intención de proteger a las personas consideradas como vulnerables de la familia [niños, jóvenes o personas ancianas] puede ocasionar el efecto contrario, es decir, generarles más preocupación y aislamiento ${ }^{(5-7)}$.

Teniendo en cuenta las diversas investigaciones anteriormente citadas y pretendiendo seguir la misma línea, hemos elaborado un documento sencillo, práctico y útil, realizado en forma de tríptico bilingüe (catalán, castellano), en formato DIN A4, dirigido a las personas enfermas por cáncer y a sus familiares (ver anexo 12) . La idea de elaborar este documento surgió a raíz de una necesidad detectada desde hace años en el trabajo diario de nuestras Unidades de Psicooncología y Trabajo Social respecto a las dificultades en la comunicación intrafamiliar. El objetivo principal que persigue el documento es el de favorecer la comunicación entre las personas afectadas por la enfermedad y las personas mayores de su familia. También permite y propicia el abordaje de dudas, preocupaciones y reflexiones sobre la comunicación, muy frecuentemente omitidas en las consultas médicas, donde se suele priorizar la atención a la parte más orgánica de la persona.

Nos pareció interesante que la elaboración del tríptico fuera compartida entre las Unidades de Psicooncología y Trabajo Social de nuestro hospital, dado el trabajo interdisciplinar que realizamos diariamente y la motivación que ambas unidades compartimos por la atención a la familia.

Como ya hemos comentado anteriormente, el objetivo principal del tríptico es favorecer la comunicación sobre la enfermedad entre las personas recién diagnosticadas de cáncer y las personas mayores que forman parte del núcleo familiar. Así, este sencillo documento contiene una serie de recomendaciones básicas que pretenden servir de guía en un momento de gran dificultad como es el diagnóstico de una enfermedad oncológica. Las describimos a continuación:

- Mantener respeto por las personas de edad avanzada: Se invita a reflexionar sobre el respeto hacia los mayores de la familia ya que incluso en situaciones de elevada vulnerabilidad estas personas conocen muy bien y desde hace mucho tiempo a la persona afectada por la enfermedad oncológica.

- No es aconsejable infravalorar al padre o a la madre por su edad avanzada pues a menudo detectan que está sucediendo algún acontecimiento fuera de lo habitual, ya que los cambios en los patrones de comunicación no verbal (gestos, miradas, silencios...) les transmiten mucha información.

- El hecho de detectar o percibir que está ocurriendo alguna cosa a nivel familiar y no recibir ningún tipo de explicación sobre ello, a los mayores les puede angustiar mucho más que si se les explica la situación de enfermedad y se les incluye en el proceso.

- Las personas mayores necesitan estar informadas de lo que les ocurre a sus familiares y que se les tenga en cuenta. Ser partícipes del proceso de afrontamiento 
les aportará tranquilidad en contraste a los sentimientos que puede provocar la incertidumbre generada por el desconocimiento y la ignorancia.

- Tener en cuenta a las personas mayores a lo largo de la enfermedad también puede ser de gran utilidad y ayuda, pues pueden participar en las atenciones que el enfermo necesite y proporcionar un soporte que exclusivamente ellas son capaces de aportar por su experiencia de vida y por el tipo de vínculo que les une a la persona enferma.

- En el momento de dar la información es mejor hacerlo siempre que sea posible acompañado.

- Es necesario tener en cuenta que la persona enferma conoce perfectamente a la persona de edad avanzada de la familia y por lo tanto sabrá como aproximarse, como comunicarse y mantener un diálogo sincero, respetando en todo momento el ritmo necesario.

- Recordar que tras informar a los mayores hay que dejarles tiempo para digerir la situación y mantener la confianza en sus recursos para gestionar los momentos de dificultad (ellos por su veteranía han gestionado de anteriores...) y para gestionarse.

- Para compartir las ansiedades o preocupaciones sobre este tema, las personas afectadas por la enfermedad pueden dirigirse a los profesionales especializados en asesorar sobre la comunicación intrafamiliar.

Este documento se distribuyó en las salas de espera de los diferentes servicios oncológicos de nuestro centro: Servicio de Oncología Médica, Servicio de Oncología Radioterápica y planta de Hospitalización de Oncología, Hemato-Oncologia y Cuidados Paliativos. También se distribuyeron ejemplares en las consultas externas de Psicooncologia, Trabajo Social y en la consulta de enfermería del Hospital de Día, como material de soporte para los profesionales que atienden a las personas afectadas en estos espacios. Para facilitar y ampliar el acceso al documento a cuantas más personas diagnosticadas y familiares, se distribuyó también entre las diferentes Unidades de Psicooncología i Trabajo Social de todos los centros ICO de nuestra área de influencia.

De la experiencia concluimos que la creación y distribución de este tríptico ha sido ventajosa, por un lado porque ha mostrado ser de gran utilidad para las personas afectadas, así como para sus familiares, y por otro lado hemos detectado que este tríptico también supone una buena ayuda para los profesionales de enfermería que, sin ser psicólogos, afrontan cuestiones relacionadas con la comunicación planteadas por los pacientes en las primeras visitas de contacto, pues con este escrito pueden facilitar una primera orientación al paciente oncológico, así como valorar la posibilidad y necesidad de derivación a las unidades de Psicooncología y/o Trabajo Social para atender cuestiones más especializadas.

Finalmente, pretendemos reconocer y validar el derecho de las personas de edad avanzada a ser tenidas en cuenta y a ser atendidas en cualquier situación de riesgo y vulnerabilidad familiar. 


\section{Referencias bibliograficas}

1. Riquelme M. La representación del cáncer en la persona enferma y en su familia. La implicación de sus significados en la comunicación familiar. Comunitaria 2015; 9; 120-36.

2. Fuentes $\mathrm{S}$. Comunicació en càncer: Anàlisi de les preocupacions i dels recursos dels pares recent diagnosticats al comunicar la malaltia als seus fills. (Tesis doctoral) . Barcelona: Universidad Autónoma de Barcelona, 2015.

3. Sordo T, Rubio I, Peralta M, Ángeles M, i Ospina L. ¿Cómo se lo digo a mi hijo/a? Psicooncologia, 2014; 11(supl. 1): 1-83. https://doi.org/10.5209/PSIC.57082

4. Mesquida V, Seijas R, Rodríguez M. Los niños ante la pérdida de uno de los progenitores: revisión de pautas de comunicación eficaces. Psicooncologia 2015; 12: 417-29. https:// doi.org/10.5209/rev_PSIC.2015.v12.n2-3.51019

5. Fernández Hawrylak M, Hernández Maíllo L, Martínez García I. Comunicación intrafamiliar y cáncer de mama. Psicooncología 2018;15:103-18. https://doi.org/10.5209/ PSIC.59180.1.

6. Díaz V, Ruiz M, Flórez C, Urrea Y, Córdoba V, Arbeláez C, Rodríguez D. Impacto familiar del diagnóstico de muerte inminente. Rev Psicol Univ Antioquia [Internet]. 2013;5:81-4. [Acceso el 27 de febrero de 2021]. Disponible en: https://revistas.udea.edu.co/index.php/ psicologia/article/view/19686

7. Novellas A. La atención de situaciones de final de vida desde el trabajo social. Revista de Treball Social 2017; 210: 37-53. 
ANEXO 1
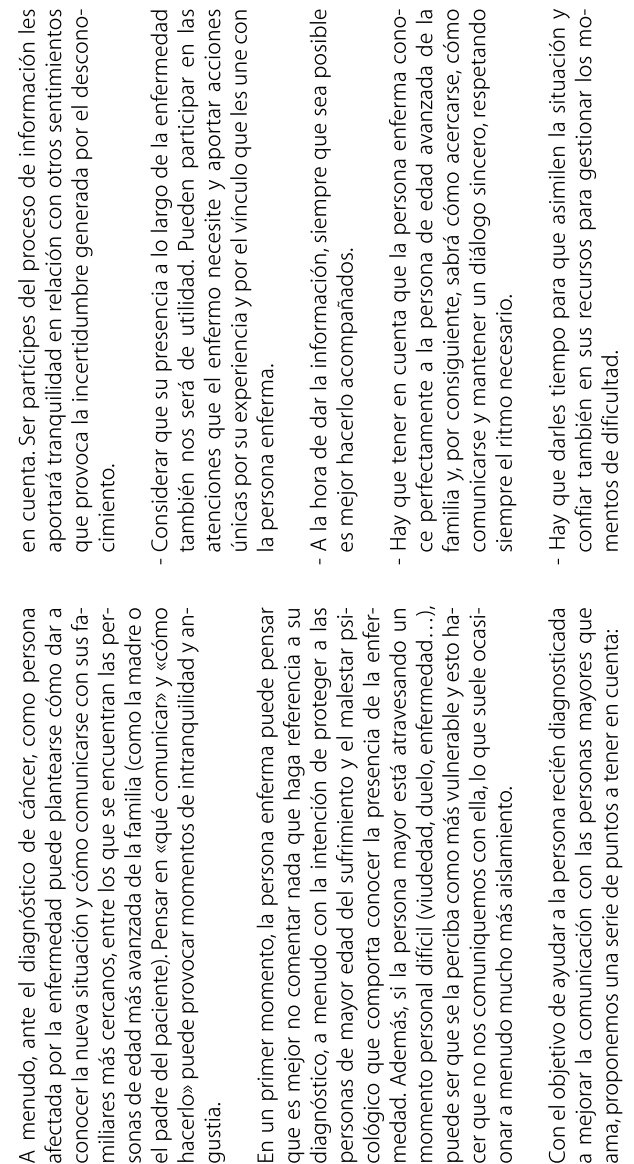

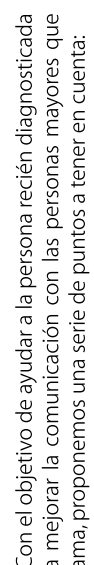

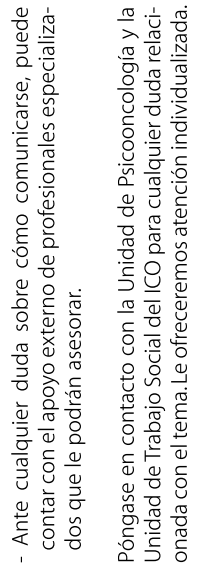

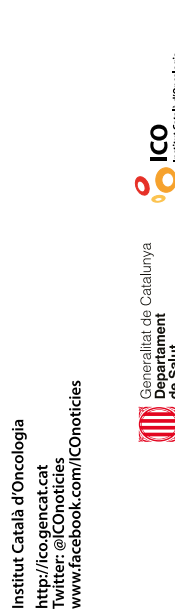

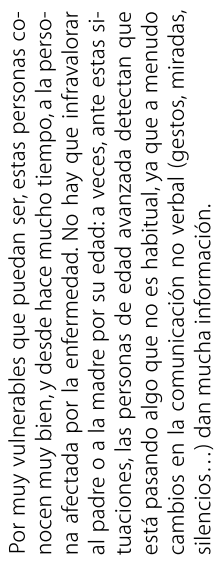

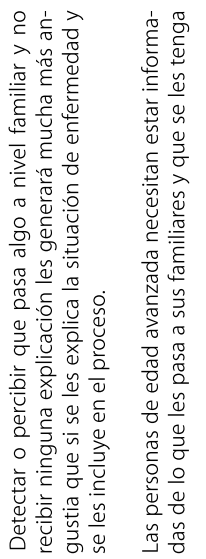

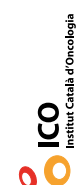

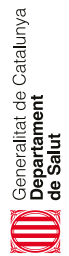

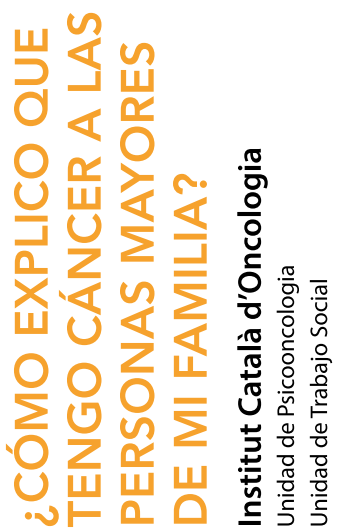
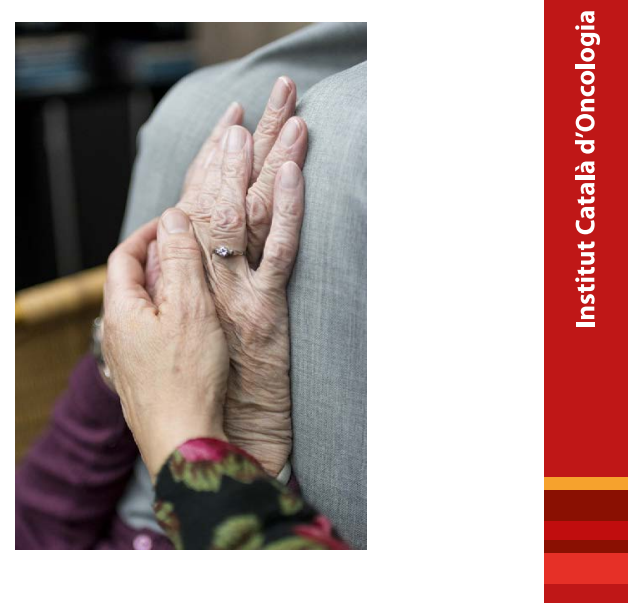